\title{
Ultrasmall superparamagnetic iron oxide (USPIO)-based liposomes as magnetic resonance imaging probes
}

This article was published in the following Dove Press journal:

International Journal of Nanomedicine

8 May 2012

Number of times this article has been viewed

\author{
Daniela Frascione ${ }^{1}$ \\ Clemens Diwoky² \\ Gunter Almer ${ }^{1,3}$ \\ Peter Opriessnig ${ }^{2}$ \\ Caroline Vonach' \\ Kerstin Gradauer \\ Gerd Leitinger ${ }^{4}$ \\ Harald Mangge ${ }^{3}$ \\ Rudolf Stollberger ${ }^{2}$ \\ Ruth Prass| ${ }^{1,5}$ \\ 'Institute of Biophysics and \\ Nanosystems Research, Austrian \\ Academy of Sciences, Graz, Austria; \\ ${ }^{2}$ Institute of Medical Engineering, \\ University of Technology, Graz, \\ Austria; ${ }^{3} \mathrm{Clinical}$ Institute for Medical \\ and Chemical Laboratory Diagnosis \\ (CIMCL), Medical University, Graz, \\ Austria; ${ }^{4}$ nstitute of Cell Biology, \\ Histology and Embryology, Medical \\ University, Graz, Austria; ${ }^{5}$ Ludwig \\ Boltzmann Institute for Lung Vascular \\ Research, Graz, Austria
}

Background: Magnetic liposomes (MLs) are phospholipid vesicles that encapsulate magnetic and/or paramagnetic nanoparticles. They are applied as contrast agents for magnetic resonance imaging (MRI). MLs have an advantage over free magnetic nanocores, in that various functional groups can be attached to the surface of liposomes for ligand-specific targeting. We have synthesized PEG-coated sterically-stabilized magnetic liposomes (sMLs) containing ultrasmall superparamagnetic iron oxides (USPIOs) with the aim of generating stable liposomal carriers equipped with a high payload of USPIOs for enhanced MRI contrast.

Methods: Regarding iron oxide nanoparticles, we have applied two different commercially available surface-coated USPIOs; sMLs synthesized and loaded with USPIOs were compared in terms of magnetization and colloidal stability. The average diameter size, morphology, phospholipid membrane fluidity, and the iron content of the sMLs were determined by dynamic light scattering (DLS), transmission electron microscopy (TEM), fluorescence polarization, and absorption spectroscopy, respectively. A colorimetric assay using potassium thiocyanate (KSCN) was performed to evaluate the encapsulation efficiency (EE\%) to express the amount of iron enclosed into a liposome. Subsequently, MRI measurements were carried out in vitro in agarose gel phantoms to evaluate the signal enhancement on T1- and T2-weighted sequences of sMLs. To monitor the biodistribution and the clearance of the particles over time in vivo, sMLs were injected in wild type mice.

Results: DLS revealed a mean particle diameter of sMLs in the range between 100 and $200 \mathrm{~nm}$, as confirmed by TEM. An effective iron oxide loading was achieved just for one type of USPIO, with an EE\% between $74 \%$ and $92 \%$, depending on the initial Fe concentration (being higher for lower amounts of $\mathrm{Fe}$ ). MRI measurements demonstrated the applicability of these nanostructures as MRI probes.

Conclusion: Our results show that the development of sMLs is strictly dependent on the physicochemical characteristics of the nanocores. Once established, sMLs can be further modified to enable noninvasive targeted molecular imaging.

Keywords: magnetic liposomes, fluorescence polarization, biodistribution, MRI contrast agent

\section{Introduction}

Liposomes are vesicular systems that are formed when phospholipids are dispersed in an aqueous solution and self-assemble into one (unilamellar) or more (oligolamellar, multilamellar) concentric bilayers surrounding an aqueous core. For several years, liposomes have occupied the center of interest because of their multiple advantages. ${ }^{1,2}$ One is their capability of encapsulating polar drugs or contrast agents in their aqueous inner core. In particular, their biocompatibility, which is defined as the quality of 
having no toxic or injurious effects on biological systems, enables them to be utilized as carriers, either for therapeutics or diagnostics in vivo. ${ }^{3-6}$

In this study, the synthesis of liposomes that offer the opportunity for targeted, noninvasive diagnosis by magnetic resonance imaging (MRI) will be described. For this purpose, iron oxide nanoparticles can be encapsulated into phospholipid vesicles to develop liposomal contrast agents (ie, magnetic liposomes [MLs] $).^{7-11}$ Here, we have focused our attention on ultrasmall superparamagnetic iron oxide nanoparticles (USPIOs), which have a hydrodynamic diameter of less than $50 \mathrm{~nm}$. In contrast to superparamagnetic iron oxide nanoparticles (SPIOs; diameters between 70 and $150 \mathrm{~nm}$ ), which are used as T2 contrast agents, USPIOs additionally show a T1 contrast when applied in moderate concentrations. ${ }^{12-14}$ More precisely, USPIOs affect the relaxivity of nearby water protons by decreasing their $\mathrm{T} 1$ and $\mathrm{T} 2$ relaxation times at nanomolar concentrations. This can be seen as increased signal intensity (hyperintense T1 effect) when applying a T1-weighted sequence, and as decreased signal intensity (hypointense T2 effect) when using T2-weighted sequences. ${ }^{15-17}$ USPIOs consist of a magnetic core that is usually coated by various materials (eg, citrate, dextran, or oleic acid) to improve the solubility and the stability of the nanoparticles in colloidal suspension. ${ }^{18,19}$ However, the coating is not always strongly retained by the core, and due to gravitational forces, USPIOs dispersed in an aqueous solution tend to aggregate and precipitate, resulting in a destabilization of the suspension. ${ }^{20}$ Moreover, the lack of an efficient coating of the magnetic cores increases their susceptibility to aging effects - which can affect the surfaces via oxidation of magnetite $\left(\mathrm{Fe}_{3} \mathrm{O}_{4}\right)$ and maghemite $\left(-\mathrm{Fe}_{2} \mathrm{O}_{3}\right)-$ resulting in a particle with completely different physicochemical and magnetic properties than expected. ${ }^{21}$ To overcome these problems, USPIOs can be captured into liposomes to protect them from both aggregation and oxidation phenomena. Even more importantly, if a high payload of iron is packed into a liposome, the resulting magnetic dipole moment will be markedly enhanced, and this will lead to a stronger MRI signal, relative to a single magnetic core. ${ }^{12,22}$ This particular feature makes MLs suitable MRI contrast agents for several biomedical applications. ${ }^{16}$ However, for in vivo applications, the circulation time of MLs in the bloodstream should be long enough to ameliorate target-ligand interaction. This goal can be achieved by steric stabilization of the liposomes (eg, by adding a poly-ethylene glycol [PEG]-based phospholipid to the lipid formulation). Lipid-anchored PEG stabilizes the liposome and prevents the rapid recognition and consequent uptake of MLs via the mononuclear phagocyte system. ${ }^{23-25}$ In addition, functionalized derivatives of PEG-lipids can be used for the convenient coupling of targeting sequences to the surface of the liposomes to achieve specific cellular recognition.

Here, we have synthesized PEG-coated stealth magnetic liposomes (sMLs) by evaluating two different types of commercially available USPIOs. We have analyzed the chemical and physical characteristics of the sMLs, including iron oxide loading capacity, encapsulation efficiency, particle size, morphology, zeta potential, and the influence of the magnetic cores on phospholipid membrane fluidity. In addition, the magnetic properties of sMLs were investigated in vitro in agarose gel phantoms and in vivo in wild type mice, following the biodistribution and the clearance of the particles over time. Accordingly, the effects of the liposomal contrast agents on both negative and positive MRI signal enhancement were evaluated with the goal of establishing an optimal formulation for the synthesis of targeted, multifunctional nanoconstructs.

\section{Material and methods Materials}

The following lipids were used for the synthesis of sMLs: 1-Palmitoyl-2-oleoyl-sn-glycero-3-phosphocholine (POPC); 1,2-distearoyl-sn-glycero-3-phosphoethanolamine-N(methoxy [polyethylene glycol]-2000) (DSPE-Peg-2000); and cholesterol $(\mathrm{CH})$ were purchased from Avanti Polar Lipids Inc (Alabaster, AL).

Iron oxides $\left(\mathrm{Fe}_{3} \mathrm{O}_{4}\right)$ were used for encapsulation in sMLs; magnetite grains coated with a polar surfactant (EMG 1500) were purchased from FerroTec Corp (Tokyo, Japan), and dextran-coated USPIOs (Molday Ion, catalog no CL30Q02-2) were acquired from BioPal, Biophysics Assay Laboratory Inc (Worcester, MA). A phospholipids assay kit was purchased from Rolf Greiner BioChemica $\mathrm{GmbH}$ (Flacht, Germany); potassium thiocyanate $(\mathrm{KSCN}) \geq 99 \%$, per analysis was acquired from Carl Roth $\mathrm{GmbH}$ and $\mathrm{Co}$ (Karlsruhe, Germany); 1-(4-trimethylammoniumphenyl)-6phenyl-1,3,5-hexatriene p-toluenesulfonate (TMA-DPH) was purchased from from Invitrogen LifeTech (Carlsbad, CA); Agar-Agar (Kobe I) was obtained from Carl Roth $\mathrm{GmbH}$ and Co KG; and isofluorane was obtained from Abbott $\mathrm{GmbH}$ (Vienna, Austria). All other chemicals were of an analytical grade or of the best grade available.

\section{Methods}

\section{Synthesis of magnetic liposomes}

sMLs were prepared using a thin lipid film rehydration method. Lipids were dissolved in organic solvent 
(chloroform:methanol $=2: 1 \mathrm{v} / \mathrm{v}$ ) and mixed in a molar ratio of 3:2:0.15= POPC:CH:DSPE-Peg-2000 in a round-bottom flask. The solution was evaporated until the point of dryness under a stream of nitrogen, and the dry lipid film was left in a vacuum chamber overnight. For iron oxide nanoparticles, we used either an aqueous colloidal suspension of dextran-coated USPIOs (Molday Ion from BioPal; hydrodynamic diameter of $30 \mathrm{~nm}$; concentration of $10 \mathrm{mg} \mathrm{Fe} / \mathrm{mL}$ in water) or dry polar-coated magnetite grains (EMG 1500 from FerroTec Corp; nominal particle diameter of $10 \mathrm{~nm}$; no specifications for the coating material were provided by the manufacturer). As the latter particles were not water soluble, we prepared stock solutions containing $4.3 \mathrm{mg}$ of $\mathrm{Fe} / \mathrm{mL}$ in butanol.

In the case of Molday-Ion USPIOs, sMLs were prepared by rehydrating the lipid films (10 $\mathrm{mg}$ of phospholipids) with $1 \mathrm{~mL}$ of a HEPES buffer (20 mM of HEPES, $150 \mathrm{mM}$ of $\mathrm{NaCl}$; pH 7.4) containing different amounts of USPIOs, which corresponded to iron concentrations of $0.5,1.0,2.0$, and $3.0 \mathrm{mg} \mathrm{Fe} / \mathrm{mL}$. During rehydration, the particles were held for 1 hour at $40^{\circ} \mathrm{C}$ and vortexed intermittently to resuspend the lipid film completely. Subsequently, five cycles of freeze and thaw treatment were performed to improve the encapsulation of the magnetic cores into liposomes. The size of the sMLs was adjusted by extrusion through $200 \mathrm{~nm}$ polycarbonate membrane filters (Millipore, Billerica, MA) using a LiposoFast pneumatic extruder (Avestin Inc, Ottawa, Canada). In the case of EMG-1500 USPIOs, the butanolic solution was added to the lipid film, dried under a stream of nitrogen, and resuspended with a HEPES buffer, as described above. Since EMG-1500 USPIOs stuck to the filter membrane, the extrusion process did not work correctly, and we had to apply a sonication technique to increase homogeneity. We used an AA Sonics Vibra-Cell Ultrasonicator (Sonics and Materials Inc, Newtown, CT), which we operated for 30 minutes at 130 Watts (30\% amplitude with a pulse sequence of 30 seconds on and 2 seconds off). Control liposomes were prepared as described above, but hydration was performed with $1 \mathrm{~mL}$ of HEPESbuffered saline in the absence of magnetite.

\section{Purification of stealth magnetic liposomes}

Nonentrapped Molday-Ion USPIOs were separated by ultrafiltration. A volume of about $1 \mathrm{~mL}$ of sMLs was applied in a Vivaspin 6 tube (MWCO $1000 \mathrm{kD}$, polyethersulfone membrane; Sartorius Stedim Biotech, Göttingen, Germany) and centrifuged at $4^{\circ} \mathrm{C}$ for 40 minutes at $6000 \times \mathrm{g}$ with a fixangle rotor, using a table centrifuge (Sigma 3 K18; SigmaAldrich, St Louis, MO). Washing was performed with a HEPES buffer; EMG-1500-loaded sMLs were purified by centrifugation for 15 minutes at $1000 \times \mathrm{g}$, and the supernatant was collected.

\section{Chemical characterization of stealth magnetic liposomes \\ Assay of magnetite}

The amounts of iron were determined photometrically using KSCN as follows: Aliquots of $20 \mu \mathrm{L}$ of liposomal solutions before and after purification, as well as from the filtrate containing the unloaded iron, were mixed with $5 \mu \mathrm{L}$ of Triton X-100 [1\% (v/v) in the final solution] to break the liposomes and release the USPIOs. A volume of $0.225 \mathrm{~mL}$ of concentrated $\mathrm{HCl}(37 \%)$ was then added to the samples to ionize the iron oxide crystal core and liberate the iron in its ferric state. The samples were incubated for a few minutes with $0.250 \mathrm{~mL}$ of a $40 \mathrm{mM} \mathrm{KSCN}$ aqueous solution. The product of the reaction between the anion ( $\mathrm{SCN}$ ) and the $\mathrm{Fe}^{3+}$ was a red colored complex - pentaaqua(thiocyanate-N) $\mathrm{Fe}(\mathrm{III}),\left[\mathrm{Fe}(\mathrm{NCS})\left(\mathrm{H}_{2} \mathrm{O}\right)_{5}\right]^{2+}-$ whose absorbance $(\mathrm{ABS})$ at $480 \mathrm{~nm}$ was read using a Hitachi model V-2000 double-beam UV-vis spectrophotometer (Tokyo, Japan). An aqueous solution of $\mathrm{Fe}_{3} \mathrm{O}_{4}$ in $\mathrm{HCl}$ :HEPES $=1: 1(\mathrm{v} / \mathrm{v})$ was used to record a calibration curve.

\section{Assay of phospholipids}

The amount of POPC in sMLs was determined by an enzymatic colorimetric test, as described by the manufacturer (DiaSys Diagnostic Systems GmbH, Holzheim, Germany). Samples $(10 \mu \mathrm{L})$ - before and after centrifugation - were mixed with Triton X-100 and incubated with enzymatic reagents for 5 minutes at $37^{\circ} \mathrm{C}$, and the $\mathrm{ABS}$ was read at $570 \mathrm{~nm}$. The fractions containing the free USPIOs after purification were also analyzed to verify the absence of lipids in these specimens. The calibration curve was performed by measuring the ABS value of a phospholipid standard contained in the kit under the same conditions.

\section{Determination of encapsulation efficiency (EE\%)}

This value designates the percentage of iron loaded into a liposome, and is given by the ratio between the iron concentration in magnetic liposomes before and after purification $(\mathrm{EE} \%$ is expressed as $\mathrm{g} \mathrm{Fe} / \mathrm{mol} \mathrm{POPC} \times 100)$.

\section{Physical characterization of stealth magnetic liposomes}

Particle size and zeta potential

The particle sizes of free USPIOs (EMG 1500 and Molday Ion), control liposomes, and sMLs were analyzed by DLS 
using a Zetasizer 3000HS (Malvern Instruments, Herrenburg, Germany). To check the stability of Molday-Ion sMLs, we also measured the sizes of the liposomes 1 week after sample preparation. The zeta potential of Molday-Ion USPIOs and Molday-Ion sMLs was measured in a 4-(2-hydroxyethyl)1-piperazineethanesulfonic acid (HEPES) buffer using a Zetasizer Nano ZS (Malvern Instruments).

\section{Particle visualization}

Liposomes and USPIOs were visualized by transmission electron microscopy (TEM) using a Zeiss EM 902 transmission electron microscope (Oberkochen, Germany) at $80 \mathrm{kV}$ acceleration voltage. Digital images were recovered with a Proscan slow scan charge-coupled device camera (IR1K, Moorenweis, Germany) at $1000 \mathrm{~K} \times 1000 \mathrm{~K}$ resolution. To mark the liposomes, a single droplet negative staining technique was performed as follows: a drop of each specimen $(10 \mu \mathrm{L})$ was placed on a Pioloform-coated carbon evaporated copper grid (Plano GmbH, Wetzlar, Germany). After 1 minute, the excess sample was removed with filter paper and replaced by $10 \mu \mathrm{L}$ of staining solution consisting of $2 \%$ ammonium molybdate and $1 \%$ trehalose as a preservative $(\mathrm{pH}$ 7.4). Finally, the samples were allowed to air dry for TEM visualization with a magnification of $\times 30000$.

\section{Fluorescence polarization (FP)}

TMA-DPH is a diphenylhexatriene (DPH) derivative containing a cationic trimethylammonium substituent. This fluorescent probe has the special property of being able to intercalate into the liposomal membrane, allowing the assessment of specific alterations in the fluidity of lipid assemblies. $^{26-28}$

Here, we have compared sMLs to empty liposomes and empty liposomes just incubated with USPIOs, respectively. Additionally, we have investigated the impact of $\mathrm{CH}$ or $\mathrm{PEG}$ lipids on fluorescence polarization, using either POPC/DSPEPeg-2000 = 3/0.15 $(\mathrm{mol} / \mathrm{mol})$ or POPC $/ \mathrm{CH}=3 / 2(\mathrm{~mol} / \mathrm{mol})$ formulations (10 mg/mL phospholipids), both loaded and unloaded with magnetite. TMA-DPH was dissolved in ethanol $(10 \mathrm{mM})$ and further diluted with a HEPES buffer to give a concentration of $200 \mu \mathrm{M}$. TMA-DPH stock was added to the prediluted liposomal solutions $(600 \mu \mathrm{M})$ to achieve a final fluorophore concentration in the quartz cuvette of $2 \mu \mathrm{M}$. The samples were then enveloped in aluminum foil, incubated at room temperature for 30 minutes, and accurately vortexed in the absence of direct light. Fluorescent measurements were carried out at $25^{\circ} \mathrm{C}$ using a Fluoromax-3 spectrofluorometer (Jobin Yvon, Horiba, Germany) at excitation/emission wavelengths set to $340 / 425 \mathrm{~nm}$ and $1.4 \mathrm{~nm}$ excitation and emission slit widths. The equation applied to calculate the anisotropy $(<\mathrm{r}\rangle)$ of the system, which is a measure of molecular mobility, was as follows:

$$
<\mathrm{r}>=\mathrm{I}_{\mathrm{VV}}-\mathrm{I}_{\mathrm{VH}} / \mathrm{I}_{\mathrm{VV}}+2 \mathrm{I}_{\mathrm{VH}}
$$

where $I_{V V}$ is the vertically polarized excitation component and indicates the position of the excitation polarizer, while $I_{V H}$ corresponds to the vertical and horizontal emission components and denotes the emission polarizer location. The intensity data were corrected by subtracting the contribution given by the scattering of blank samples without a fluorophore in the bilayer.

\section{Magnetic resonance imaging in vitro measurements}

MRI was performed with a Magnetom Tim Trio 3T machine (Siemens, Erlangen, Germany) using an 8-CH multipurpose coil (NORAS MRI products GmbH, Höchberg, Germany).

The saturation magnetizations of sMLs $(0.8 \mathrm{mg}$ POPC $/ \mathrm{mL}$ ) and free USPIOs - applied at the same iron concentrations as the sMLs - were measured using a susceptometry method, as described by Bowen et al. ${ }^{29}$

For representative T1- and T2-weighted images, freshly prepared Molday-Ion sMLs and EMG-1500 sMLs were embedded in uniform gel suspensions $(1 \% \mathrm{w} / \mathrm{w}$ agarose, $1.2 \mathrm{mg}$ POPC $/ \mathrm{mL}$ ) and placed into a spherical gel phantom in order to avoid interference produced by macroscopic B0 inhomogeneities.

With the strong T2 and restrictions and the shortest possible echo time in mind, we used an inversion recovery Rapid Acquisition with Relaxation Enhancement to show the T1 effect. T1-weighted images were acquired with an inversion time of TI $=50 \mathrm{~ms}, \mathrm{FOV}=70 \times 100 \mathrm{~mm}$, matrix $=180 \times 256$, slice thickness $=5 \mathrm{~mm}, \mathrm{TR}=5000 \mathrm{~ms}, \mathrm{TE}=11 \mathrm{~ms}, \mathrm{TF}=5$, BW/pixel: $349 \mathrm{~Hz}, \mathrm{NSA}=1$. T2-weighted images were acquired with a CPMG pulse sequence with echo times of $\mathrm{TE}=13 / 26 / 39 / 52 / 65 \mathrm{~ms}, \mathrm{FOV}=81 \times 100$, matrix $=166 \times 256$, slice thickness $=5 \mathrm{~mm}, \mathrm{TR}=2000 \mathrm{~ms}, \mathrm{BW} /$ pixel: $425 \mathrm{~Hz}$, $\mathrm{NSA}=1$. In order to better visualize the differences in $\mathrm{T} 2$ enhancement and to avoid complete signal loss, the first echo was chosen.

\section{Animal model and ethics}

All animal experiments were carried out in accordance with the approvals of the Ministry of Science and Research, Austria. Nine wild type mice of random sex (Medical University of Vienna, Austria) that weighed between $29.3 \mathrm{~g}$ and $33.7 \mathrm{~g}$ 
were used in the biodistribution studies: three sham mice were used as control, while the other six were separated into groups of three, either for the injection of Molday-Ion sMLs (between $170 \mu \mathrm{L}$ and $190 \mu \mathrm{L}$, corresponding to $50 \mu \mathrm{mol} \mathrm{Fe} /$ $\mathrm{kg}$ body weight) or free USPIOs (equivalent in iron content to the sMLs). Before injection into the retro-orbital sinus with a 27 gauge needle, the mice were placed in an induction chamber and narcotized with $5 \%(\mathrm{v} / \mathrm{v})$ isofluorane (ISO), 1.5 bar/oxygen till gasping. Eight days after injection, mice were sacrificed via an ISO overdose; then, about $250 \mu \mathrm{L}$ of total blood was collected by cardiac puncture, and the circulation was immediately rinsed with phosphate buffer saline PBS ( $\mathrm{pH} 7.4$ ) for 15 minutes by heart perfusion. The organs (liver and kidneys) were accurately removed, fixed for 30 minutes in $4 \%(\mathrm{v} / \mathrm{v})$ paraformaldehyde in PBS $(\mathrm{pH}$ 7.4), rinsed with PBS, shock-frozen, and stored at $-80^{\circ} \mathrm{C}$ for further analysis.

\section{Inductively coupled plasma optical emission spectrometry}

Blood samples and weighed portions of liver and kidneys underwent microwave-assisted digestion in an acidic mixture ( $3 \mathrm{~mL}$ of $\mathrm{HNO}_{3}, 1 \mathrm{~mL}$ of $\mathrm{HCl}, 2 \mathrm{~mL}$ of water) until complete decomposition, using an Anton Paar Multiwave 3000 system (Anton Paar GmbH, Graz, Austria). The iron content was subsequently determined via inductively coupled plasma optical emission spectrometry (ICP-OES) with a Spectro Ciros Vision Optical emission spectrometer instrument (Spectro GmbH, Kleve, Germany) at $1400 \mathrm{~W}$ RF power; $12.5 \mathrm{~L} / \mathrm{min}$ Argon (Ar) outer gas flow; $0.6 \mathrm{~L} / \mathrm{min}$ Ar intermediate gas flow; $0.86 \mathrm{~L} / \mathrm{min}$ nebulizer gas flow; scot type spray chamber; and cross-flow nebulizer. The instrument was calibrated with iron solutions ranging from $0 \mathrm{mg} \mathrm{Fe} / \mathrm{L}$ to $5.0 \mathrm{mg} \mathrm{Fe} / \mathrm{L}$, and Scandium (Sc) was employed as an internal standard measurement criterion for four replicates at $24 \mathrm{~s}$ integration; $\mathrm{Fe}$ (II) 238,204 nm was used.

\section{Magnetic resonance imaging in vivo analysis}

For the distribution study, the relative changes in the relaxation times of muscle, liver, and kidneys were quantified by quantitative relaxation time analysis after injection of either Molday-Ion sMLs $(n=3)$ or free Molday-Ion USPIOs $(n=3)$ at a dose of $50 \mu \mathrm{mol} \mathrm{Fe} / \mathrm{kg}$ body weight. Measurements were acquired at different time points ( 0 minutes, 5 minutes, 5 hours, 12 hours, 24 hours, and 7 days post injection).

In vivo $\mathrm{T} 1$ and $\mathrm{T} 2 *$ relaxation maps were acquired on the same system that was applied for the in vitro phantom studies, using the 8-CH multipurpose coil for high signal-to-noise ratio-whole body coverage of the mice. For the T1 measurement, an inversion-recovery turbo-spin-echo pulse sequence was used: $\mathrm{FOV}=40 \times 80 \mathrm{~mm}$, matrix $=64 \times 128$, slice thickness $=1.5 \mathrm{~mm}, \mathrm{TR}=5000 \mathrm{~ms}, \mathrm{TE}=9.5 \mathrm{~ms}, \mathrm{TF}=7$, BW/pixel: $349 \mathrm{~Hz}, \mathrm{TI}=50 / 200 / 800 / 2000 \mathrm{~ms}, \mathrm{NSA}=3$. T2* was acquired with a $2 \mathrm{D}$ multi-echo gradient-echo sequence of 4 echoes, with the following parameters: echo spacing of $5.5 \mathrm{~ms}$, first echo $=3.9 \mathrm{~ms}, \mathrm{FOV}=40 \times 80$, matrix $=96 \times 192$, slice thickness $=1.5 \mathrm{~mm}, \mathrm{TR}=261 \mathrm{~ms}, \mathrm{BW} /$ pixel: $491 \mathrm{~Hz}$, $\mathrm{NSA}=20$

\section{Statistical analysis}

Values were expressed as mean \pm standard deviation (SD). Student's $t$-test was performed to identify significant differences between samples, and $P$ values $\leq 0.01$ were assumed to be statistically significant. All tests were performed using the statistical and process management software MINITAB (v13.0; State College, PA).

\section{Results and discussion}

\section{Stealth magnetic liposome purification and encapsulation efficiency}

Immediately after extrusion/sonication, nonenclosed Molday-Ion and EMG-1500 USPIOs were removed by ultrafiltration or centrifugation, respectively. Figure 1A illustrates the aggregation and sedimentation behavior observed for EMG-1500 USPIOs in colloidal suspensions of sMLs. In contrast, Molday-Ion USPIOs were perfectly dispersed in an aqueous solution without showing any aggregation or separation from the liposomal phase after incorporation. A brownish homogenous dispersion of purified Molday-Ion sMLs was obtained (Figure 1B).

Once sMLs were recovered and free of contaminated external magnetite, their iron content was determined by a colorimetric test using $\mathrm{KSCN}$, and encapsulation efficiency

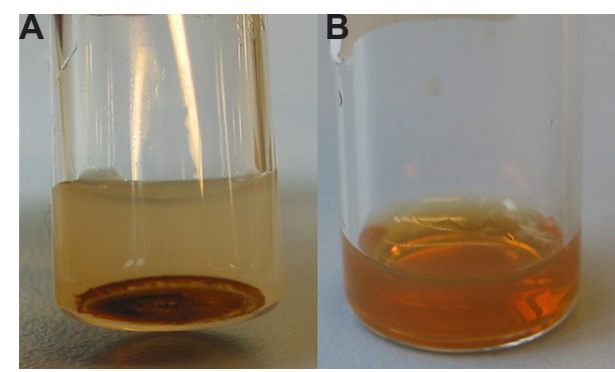

Figure I Pictures of (A) sonicated liposomes loaded with polar-coated EMG-I500 USPIOs and (B) extruded sMLs encapsulating dextran-coated Molday-lon USPIOs. Note: Photographs were taken a few hours after purification, and samples were kept at room temperature.

Abbreviation: USPIOs, ultrasmall superparamagnetic iron oxides. 
Table I Iron quantification via colorimetric assay using KSCN and encapsulation efficiency estimation of purified Molday-lon sMLs

\begin{tabular}{lccc}
\hline $\begin{array}{l}\text { Initial iron concentration for } \\
\text { sML synthesis (mg Fe/mL) }\end{array}$ & $\begin{array}{l}\text { Iron concentration of sMLs before } \\
\text { purification (g Fe/mol POPC) }\end{array}$ & $\begin{array}{l}\text { Iron concentration of sMLs after } \\
\text { purification (g Fe/mol POPC) }\end{array}$ & $\begin{array}{l}\text { Encapsulation } \\
\text { efficiency* (\%) }\end{array}$ \\
\hline 0.5 & $57.4 \pm 2.6$ & $53.1 \pm 3.8$ & $92 \pm 3.3$ \\
1.0 & $119.3 \pm 8.9$ & $89.6 \pm 4.6$ & $75 \pm 1.8$ \\
2.0 & $247.4 \pm 19.6$ & $205.7 \pm 22.1$ & $83 \pm 10.4$ \\
3.0 & $343.1 \pm 46.0$ & $252.6 \pm 38.4$ & $74 \pm 4.9$ \\
\hline
\end{tabular}

Notes: *Percentage of encapsulation efficiency was calculated from the ratio of the iron concentration in sMLs before and after purification (EE\% expressed as $g$ Fe/mol $\mathrm{POPC} \times 100)$. Values $( \pm \mathrm{SD})$ are the mean of three experimental evaluations.

Abbreviations: EE\%, encapsulation efficiency; POPC, I-Palmitoyl-2-oleoyl-sn-glycero-3-phosphocholine; sMLs, stealth magnetic liposomes.

was calculated. The corresponding data are summarized in Table 1.

For Molday-Ion sMLs, we found that the EE\% decreased with increasing quantities of USPIOs that were used for loading. Additionally, we observed the highest encapsulation efficiency of $92 \%$ for the sample with the lowest initial iron concentration of $0.5 \mathrm{mg} \mathrm{Fe} / \mathrm{mL}$. This is in agreement with the results obtained by Sabaté et $a l,{ }^{10}$ who also showed that the $\mathrm{EE} \%$ is inversely proportional to the initially applied iron content. However, even for the sample with the highest initial iron concentration ( $3 \mathrm{mg} \mathrm{Fe} / \mathrm{mL}$ ), an EE\% above $70 \%$ was achieved. Regardless of this fact, the absolute amount of iron that could be incorporated into liposomes was higher using higher initial amounts of USPIOs. This finding is in accordance with results reported by Skouras et al. ${ }^{30}$ Further increases in iron concentration (above $3 \mathrm{mg} \mathrm{Fe} / \mathrm{mL}$ ) did not improve the absolute yield (data not shown).

Additionally, we tested the stability of Molday-Ion USPIO sMLs 1 week after preparing and measuring the EE\%. We found $\mathrm{EE} \%$ values of $42 \%, 72 \%, 63 \%$, and $65 \%$ for samples having initial iron concentrations of $0.5,1.0,2.0$, and 3.0, respectively, indicating that the USPIOs become released over time.

Effective encapsulation was also confirmed by zeta potential measurements. Free Molday-Ion USPIOs showed a surface charge potential of $-6.84 \mathrm{mV} \pm 0.007 \mathrm{mV}$, which changed to $-4.41 \mathrm{mV} \pm 0.007 \mathrm{mV}$ for Molday-Ion sMLs. This value was very similar to that of empty liposomes, which had a potential of $-4.12 \mathrm{mV} \pm 1.10 \mathrm{mV}$.

Varying results were obtained for EMG-1500 sMLs, depending on the amount of nonentrapped iron after centrifugation. This value was extremely high, and only very low encapsulation efficiencies of $3 \%$ and $18 \%$ were accomplished using initial iron concentrations of 3.0 and $0.5 \mathrm{mg} \mathrm{Fe} / \mathrm{mL}$, respectively. Nevertheless, the highest EE\% was again achieved for the lowest initial quantity of iron used.

The deviating behavior of the two commercially available USPIOs, with respect to encapsulation, could be due to differences in the structure of the crystal bulk. Different methods of synthesis, reaction conditions, or a different $\mathrm{Fe}^{2+}: \mathrm{Fe}^{3+}$ ratio can generate variable magnetic cores of various sizes that contain one or more iron oxides. ${ }^{31}$ Moreover, the type, arrangement, and efficiency of the coating material on the iron oxide nanocores could modulate not only the magnetic properties and the relaxivity values of the nanoparticles, but also their stability and solubility properties, thereby influencing entrapment efficacy.

\section{Diameter and morphology of ultrasmall superparamagnetic iron oxides and stealth magnetic liposomes}

DLS experiments revealed an average hydrodynamic diameter for the dextran-coated Molday-Ion USPIOs of $23.8 \mathrm{~nm} \pm 0.4 \mathrm{~nm}$ and a polydispersity index (PDI) of $0.20 \pm 0.01(\mathrm{n}=3)$. These values are in line with data provided by the manufacturer. Extruded Molday-Ion-loaded sMLs and empty control liposomes exhibited mean hydrodynamic diameters between $160 \mathrm{~nm}$ and $170 \mathrm{~nm}(\mathrm{PDI}=0.10 \pm 0.01$ ). Notably, the average diameter size of sMLs was not significantly influenced by increasing the initial amount of USPIOs, and the particle size remained constant for at least 1 week of storage at room temperature.

In contrast, the size distribution observed for the polarcoated EMG-1500 USPIOs was very broad. The EMG-1500 USPIOs were highly heterogeneous in colloidal suspension and revealed diameters ranging from $100 \mathrm{~nm}$ to $1000 \mathrm{~nm}$ with a PDI value of $0.8 \pm 0.2$. This size - in terms of inhomogeneity is most probably the reason why EMG-1500 USPIOs could not be sufficiently incorporated into liposomes. After sonication, EMG-1500-loaded sMLs revealed a hydrodynamic diameter between $100 \mathrm{~nm}$ and $130 \mathrm{~nm}$ (PDI $=0.1 \pm 0.005$ ). A similar size distribution was found for sonicated empty liposomes that we used as the control.

To visualize USPIOs, we used TEM (Figure 2); with TEM, we could also observe the iron oxide grains (dark spots), which were tightly packed within Molday-Ion-loaded sMLs, whereas the liposomes were observed as closed quasi-spherical struc- 

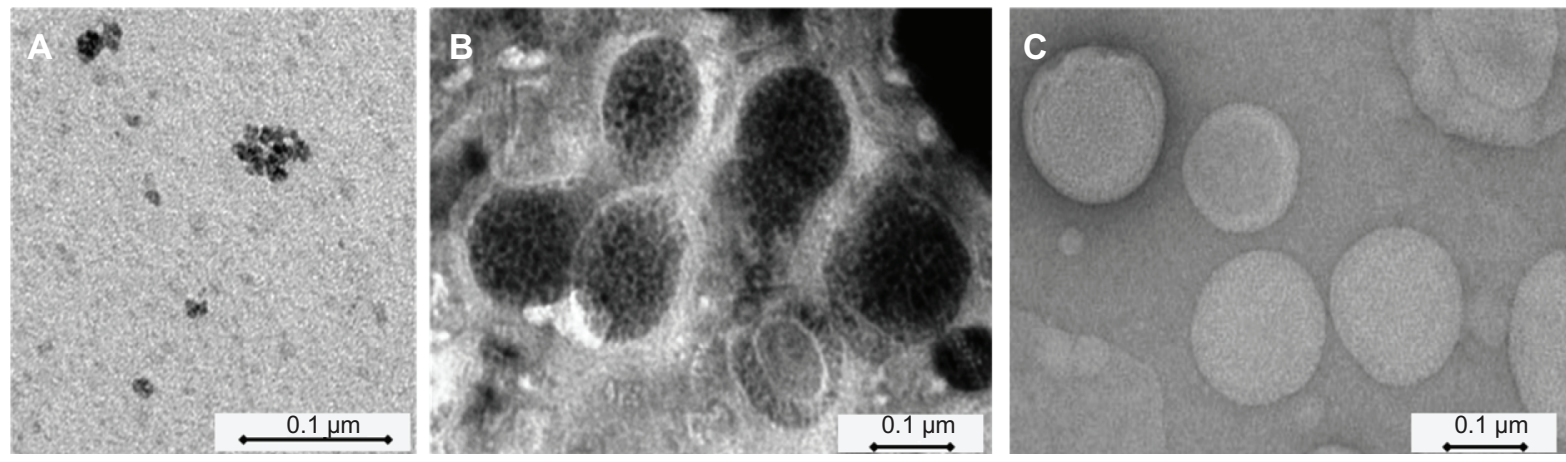

Figure 2 TEM micrographs of (A) dextran-coated Molday-lon USPIOs ( $1.7 \mathrm{mg} \mathrm{Fe} / \mathrm{mL})$ in an aqueous buffer directly observed under the microscope; (B) sMLs loaded with Molday-Ion USPIOs at a final iron concentration of $1.7 \mathrm{mg} \mathrm{Fe} / \mathrm{mL}$ (after extrusion and purification); and (C) extruded control liposomes without magnetite.

Abbreviations: sMLs, stealth magnetic liposomes; TEM, transmission electron microscope; USPIOs, ultrasmall superparamagnetic iron oxides.

tures, most of which had a diameter of less than $200 \mathrm{~nm}$. Due to the poor performance of EMG-1500-loaded liposomes, they were not further investigated by TEM.

\section{Fluorescence polarization}

To investigate whether the incorporation of Molday-Ion USPIOs in liposomes has any influence on phospholipid bilayer properties, we performed anisotropy measurements to monitor lipid membrane fluidity.

To this end, the fluorescence polarization of the amphiphilic fluorophore TMA-DPH - which preferentially locates close to the polar head group region of the lipid bilayer and probes the water-lipid interface - was recorded. We found a significant increase in the $\langle\mathrm{r}\rangle$ values from $0.241 \pm 0.003$ to $0.275 \pm 0.010(P<0.01)$ by comparing empty liposomes with purified sMLs that contained a final iron concentration of $1.7 \mathrm{mg} / \mathrm{mL}$. In contrast, incubation of preformed empty liposomes with Molday-Ion USPIOs with the same iron concentration of $1.7 \mathrm{mg} / \mathrm{mL}$ did not show a notable shift of the anisotropy value $(<\mathrm{r}>=0.248 \pm 0.002)$, compared to empty control liposomes. These data suggest that USPIOs primarily interact with the lipid membrane when they are entrapped into the liposomes. One possible explanation for this behavior is that high concentrations of USPIOs are confined to a small volume of water inside the liposomes and most probably interact with the hydrophilic phospholipid head groups in the inner leaflet of the bilayer, causing rigidification of the bilayer itself. Further evidence in support of this notion comes from experiments on formulations without $\mathrm{CH}$ or PEG lipids. As expected, these formulations were more fluid than our lead formulation that contained both DSPEPeg-2000 and $\mathrm{CH}$, but again, the anisotropy value increased upon the incorporation of USPIOs (see Table 2). In contrast, when dextran-coated USPIOs are freely dispersed in water, they have no impact on lipid membranes.

\section{Magnetic resonance imaging in vitro and in vivo}

In vitro measurements revealed that the magnetization curves determined for free USPIOs and Molday-Ion sMLs have similar shapes (Figure 3 ). In this diagram, the values for saturation magnetization are reported in $\mathrm{emu} / \mathrm{cm}^{3}$, as a function of the Fe concentration $(\mathrm{mg} / \mathrm{mL})$. The similar behavior of the magnetization curves demonstrates that the superparamagnetic properties of the iron oxide nanoparticles are preserved after encapsulation in liposomes.

Next, we compared the positive and negative signal enhancement - visualized on T1- and T2-weighted images in agarose gel phantoms - which contained either Molday-Ion or EMG-1500 USPIO-loaded liposomes.

For this purpose, we used sMLs that were freshly prepared at the same lipid concentration, but with increasing amounts of iron. Due to the different encapsulation efficiencies, the actual concentration of iron in sMLs was lower, especially for the EMG-1500 sMLs. These discrepancies in iron concentrations

Table 2 Anisotropy values $(<r>)$ of empty and Molday-lonloaded sMLs of different lipid compositions

\begin{tabular}{lllr}
\hline $\begin{array}{l}\text { Liposome } \\
\text { formulation }\end{array}$ & $\begin{array}{l}\text { Lipid molar } \\
\text { ratio }(\mathrm{mol} / \mathrm{mol})\end{array}$ & $\begin{array}{l}\mathrm{C}_{\mathrm{Fe}} \text { in sMLs } \\
(\mathbf{m g} / \mathbf{m L})\end{array}$ & Anisotropy \\
\hline $\mathrm{POPC} / \mathrm{CH} / \mathrm{Peg}$ & $3 / 2 / 0.15$ & - & $0.241 \pm 0.003$ \\
$\mathrm{POPC} / \mathrm{CH} /$ & $3 / 2 / 0.15$ & 1.7 & $0.275^{*} \pm 0.013$ \\
$\mathrm{Peg} / \mathrm{Fe}$ & & & \\
$\mathrm{POPC} / \mathrm{CH} /$ & $3 / 2 / 0.15$ & 1.7 & $0.248^{* *} \pm 0.002$ \\
$\mathrm{Peg}+\mathrm{Fe}$ & & & \\
$\mathrm{POPC} / \mathrm{Peg}$ & $3 / 0.15$ & - & $0.184 \pm 0.010$ \\
$\mathrm{POPC} / \mathrm{Peg} / \mathrm{Fe}$ & $3 / 0.15$ & 1.7 & $0.202^{*} \pm 0.004$ \\
$\mathrm{POPC} / \mathrm{CH}$ & $3 / 2$ & - & $0.203 \pm 0.003$ \\
$\mathrm{POPC} / \mathrm{CH} / \mathrm{Fe}$ & $3 / 2$ & 1.0 & $0.258^{*} \pm 0.009$ \\
\hline
\end{tabular}

Notes: Anisotropy values are presented as mean \pm SD of three experiments. *In all cases, sMLs showed a significantly increased anisotropy compared to the corresponding empty formulation $(P<0.0 \mathrm{I})$. **Subsequent incubation with free USPIOs caused no significant increase in anisotropy $(P>0.01)$.

Abbreviations: sMLs, stealth magnetic liposomes; POPC, I-Palmitoyl-2-oleoylsn-glycero-3-phosphocholine; $\mathrm{CH}$, cholesterol; Peg, polyethylene glycol; $\mathrm{C}_{\mathrm{Fe}}$, iron concentration. 


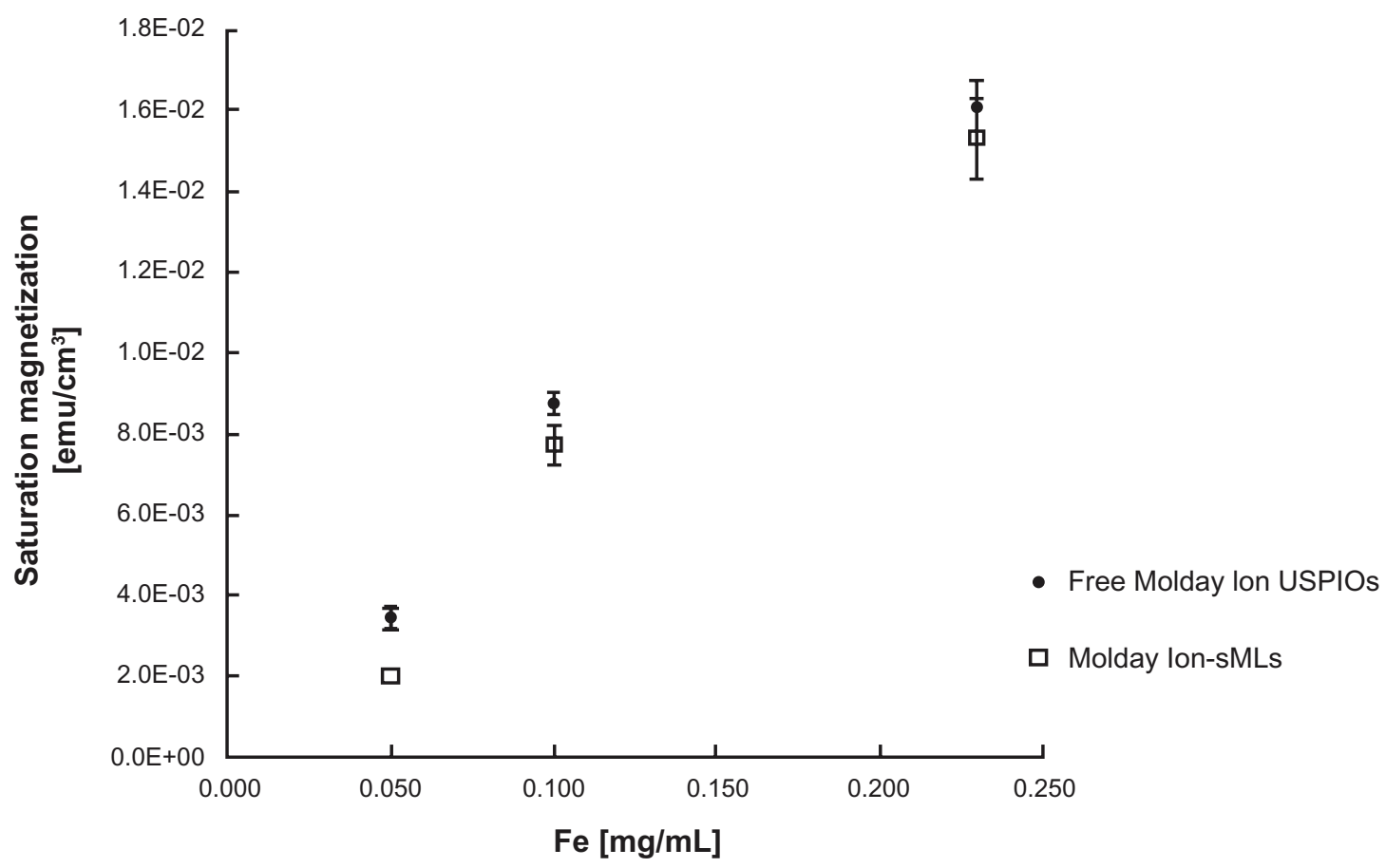

Figure 3 Saturation magnetization values $\left(\mathrm{emu} / \mathrm{cm}^{3}\right)$ of Molday-lon sMLs compared to free Molday-lon USPIOs, as a function of the Fe concentration ( $\mathrm{mg} / \mathrm{mL}$ ). Note: The points on the diagram represent the mean value of three experiments $( \pm S D)$.

Abbreviations: sMLs, stealth magnetic liposomes; USPIOs, ultrasmall superparamagnetic iron oxides.

were clearly visible in both T1 and T2 scans, as shown in Figure 4. The signal intensities of the MR scans correlated very well with the actual iron concentrations. For MoldayIon USPIO sMLs, we found that the higher the iron loading, the better the contrast enhancement; the signal recorded from the EMG-1500 sMLs was always low due to inefficient encapsulation and low effective iron concentration.

Based on the in vitro results, Molday-Ion-loaded sMLs were chosen as MRI probes for in vivo experiments, in which we studied the biodistribution of sMLs relative to free USPIO
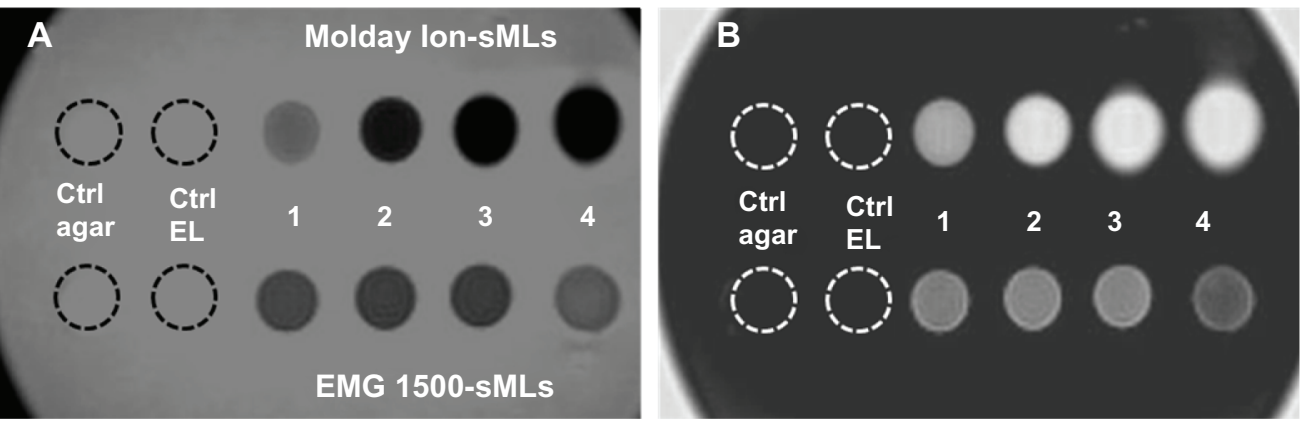

\begin{tabular}{llll}
\hline $\begin{array}{l}\text { sMLs Phantom } \\
\text { preparations }\end{array}$ & $\begin{array}{l}\text { Molday lon-/ EMG I500-sMLs } \\
\text { initial } C_{\mathrm{Fe}}(\mathbf{m g} / \mathbf{m L})\end{array}$ & $\begin{array}{l}\text { Molday lon-sMLs } \\
\text { final } C_{\mathrm{Fe}}(\mathbf{m g} / \mathbf{m L})\end{array}$ & $\begin{array}{l}\text { EMG I500-sMLs } \\
\text { final } \mathbf{C}_{\mathrm{Fe}}(\mathbf{m g} / \mathbf{m L})\end{array}$ \\
\hline $\mathrm{I}$ & 0.5 & 0.4 & 0.09 \\
2 & 1.0 & 0.8 & 0.13 \\
3 & 2.0 & 1.7 & 0.15 \\
4 & 3.0 & 2.7 & 0.08 \\
\hline
\end{tabular}

Figure 4 T2-weighted image (A) and TI-weighted scan (B) of either Molday-lon sMLs (upper part in panels A and B) or EMG-I500sMLs (lower part in panels A and B) in I\% agarose gel phantoms.

Notes: The final $\mathrm{C}_{\mathrm{Fe}}(\mathrm{mg} / \mathrm{mL})$ values correspond to the actual concentrations used for the MRI measurements shown here. Agarose gel and empty liposome are the control samples used as a reference.

Abbreviations: sMLs, stealth magnetic liposomes; $\mathrm{C}_{\mathrm{Fe}}$, iron concentration; MRI, magnetic resonance imaging; agar, agarose gel; EL, empty liposome; ctrl, control. 

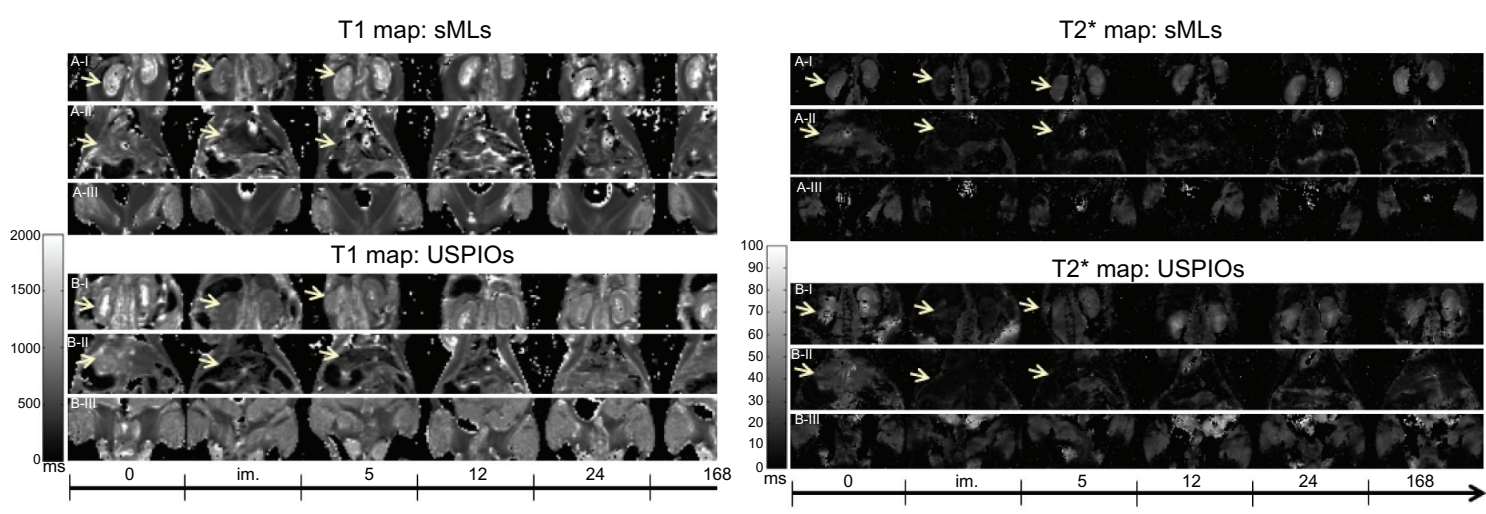

Figure 5 Behavior of the kidneys, liver, and muscle.

Notes: Panels (A-I and B-I) display the signal behavior of the kidneys; panels (A-II and B-II) display the signal behavior of the liver; and panels (A-III and B-III) display the signal behavior of the muscle over time.

Abbreviations: sMLs, steath magnetic liposomes; USPIOs, ultrasmall superparamagnetic iron oxides.

nanoparticles. $\mathrm{T} 1$ and $\mathrm{T} 2 *$ organ maps were recorded in different tissues after injection of either of the sMLs (Figure 5, panel A I-III) or free USPIOs (Figure 5, panel B I-III) in wild type mice. The images qualitatively illustrated the signal behavior of kidneys, liver, and muscle scanned before and at different time points after sample administration; sMLs showed a biodistribution similar to free Molday-Ion USPIOs.
Both induced a contrast enhancement in the kidneys and liver, lasting for at least 5 hours. This similarity in in vivo turnover rates could partially be explained by the surface characteristics of dextran-coated USPIO colloids and sMLs, both having a polymer around the core - ie, dextran in the case of USPIOs and a PEG coat in case of sMLs. The surface coating is very important in the way of prolonging the circulation time of
A

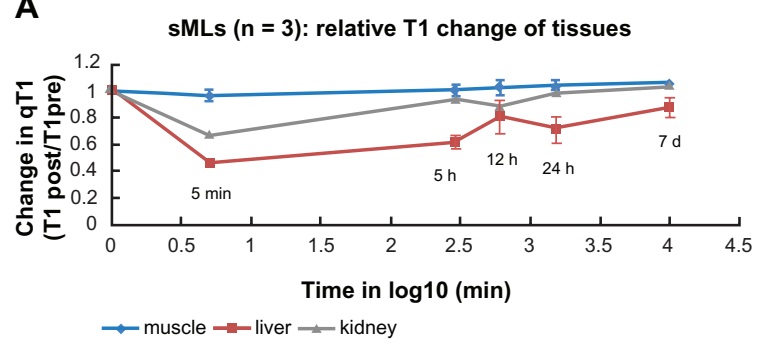

B

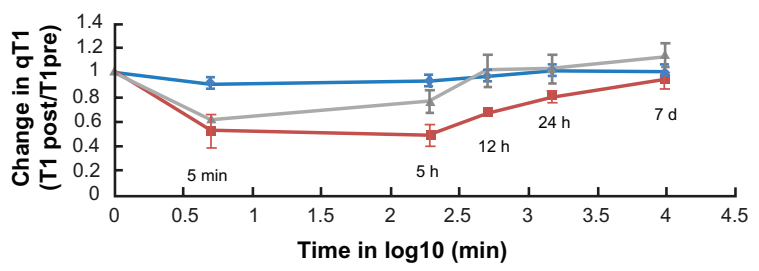

$\rightarrow$ muscle $\_$- liver $\longleftarrow$ kidney

\begin{tabular}{|l|c|c|c|}
\hline \multicolumn{1}{|c|}{$\begin{array}{c}\text { T1* } \\
\text { baseline } \\
(\mathrm{ms})\end{array}$} & Muscle & Liver & Kidney \\
\hline $\begin{array}{l}\text { sMLs-mice } \\
(\mathrm{n}=3)\end{array}$ & $875.52 \pm 43.61$ & $667.09 \pm 16.21$ & $1191.68 \pm 21.50$ \\
\hline $\begin{array}{l}\text { USPIOs-mice } \\
(\mathrm{n}=3)\end{array}$ & $924.21 \pm 21.71$ & $673.96 \pm 16.95$ & $979.29 \pm 117.38$ \\
\hline
\end{tabular}

C

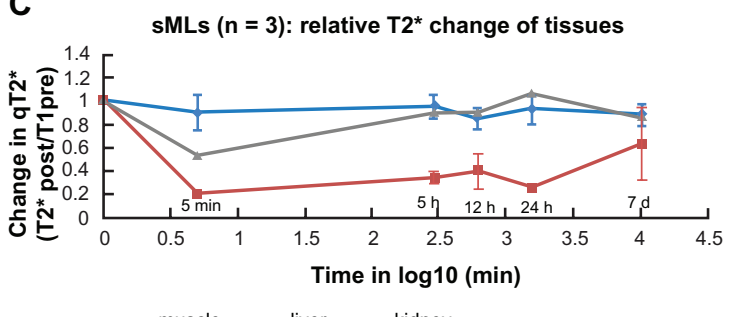

D

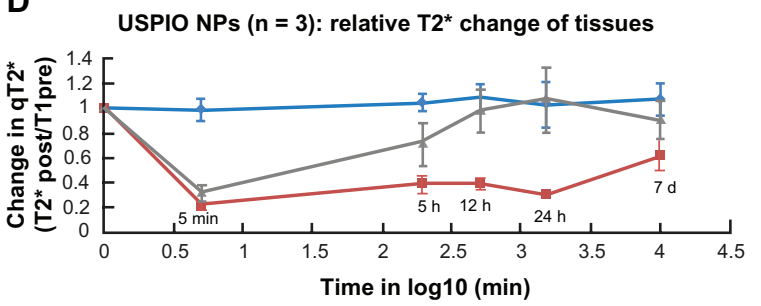

$\simeq$ muscle $\_$liver $\_$kidney

\begin{tabular}{|l|c|c|c|}
\hline $\begin{array}{l}\text { T2* } \\
\text { baseline } \\
(\mathrm{ms})\end{array}$ & Muscle & Liver & Kidney \\
\hline $\begin{array}{l}\text { sMLs-mice } \\
(\mathrm{n}=3)\end{array}$ & $20.02 \pm 1.60$ & $19.19 \pm 1.65$ & $29.10 \pm 4.05$ \\
\hline $\begin{array}{l}\text { USPIOs-mice } \\
(\mathrm{n}=3)\end{array}$ & $17.87 \pm 1.21$ & $18.71 \pm 1.37$ & $29.27 \pm 4.69$ \\
\hline
\end{tabular}

Figure 6 Relative changes in relaxation times (qTI, qT2*) of muscle, liver, and kidneys after injection of clinical doses of $50 \mu \mathrm{mol}$ Fe/Kg body weight in wild type mice. Notes: Signal changes were acquired at different time points ( 0 minutes, 5 minutes, 5 hours, 12 hours, 24 hours, 7 days) after administration of sMLs (A and C) and Moldaylon USPIOs (B and D), respectively. A strong signal reduction was observed in liver and kidneys for both sMLs and USPIOs 5 minutes after sample administration. No visible change was measured in the muscles.

Abbreviations: sMLs, stealth magnetic liposomes; USPIOs, ultrasmall superparamagnetic iron oxides; h, hours; $d$, days. 


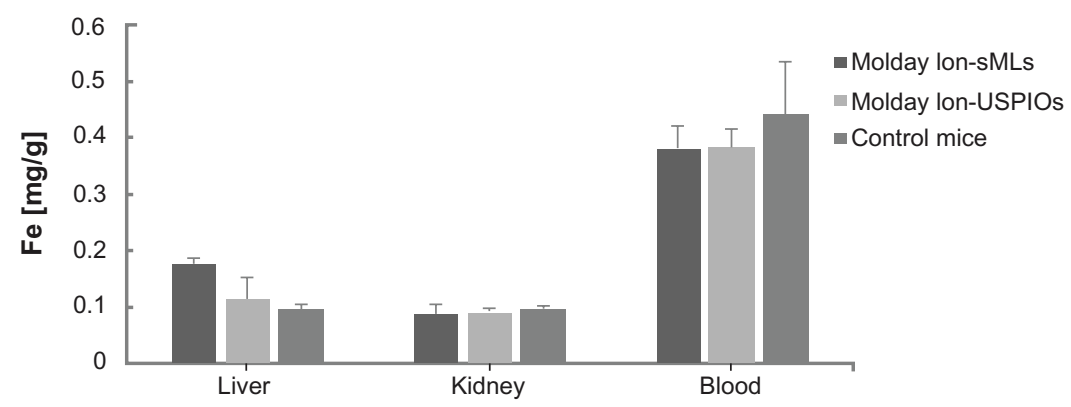

Figure 7 Quantification of iron in liver, kidneys, and blood by ICP-OES of control mice $(n=3)$ and injected mice 8 days after administration of sMLs and Molday-lon USPIOs ( $n=3$ for each).

Note: The values are means $\pm S D$.

Abbreviations: sMLs, stealth magnetic liposomes; USPIOs, ultrasmall superparamagnetic iron oxides, SD, standard deviation.

nanoparticles in the blood, thus evading the immune system more efficiently. ${ }^{23-25}$

Next, we determined the relative changes in $\mathrm{T} 1$ and $\mathrm{T} 2 *$ relaxation times via quantitative analysis. These results are reported in Figure 6, where the relative organ signal intensities (SI) are expressed as T1 post/T1 pre and T2* post/ $\mathrm{T} 2 *$ pre ratios, and the accumulation behavior of either of the sMLs (a, c) or free USPIOs (b, d) are shown.

Strong signal reduction was measured for sMLs and USPIOs in liver and kidneys on both $\mathrm{T} 1$ and $\mathrm{T} 2 *$ sequences, and 1 week later, the signal went back to the baseline level, which corresponds to the value before sample injection. In the liver, 5 minutes after injection, we found percentage reductions of $55 \%$ and $48 \%$ in the $\mathrm{T} 1$ relaxation time for sMLs and free iron oxides, respectively. After 24 hours, these values were equivalent to $30 \%$ and $20 \%$ reductions in $\mathrm{T} 1$. Also, with respect to $\mathrm{T} 2 *$ relaxation time, high reductions of $80 \%$ and $90 \%$ were achieved in the liver 5 minutes after injection of either sMLs or free Molday-Ion USPIOs, and these values were still pronounced 24 hours later $(74 \%$ for sMLs and $70 \%$ for free USPIOs). No signal reduction was detected in the muscles, indicating that the particles do not penetrate the vessel wall, and this gives the advantage of using the muscles as reference regions of signal normalization in semi-quantitative analysis.

After 7 days, sMLs and free USPIOs were completely cleared by the circulation system, as well as the kidneys, as confirmed by ICP-OES quantification (Figure 7). However, a higher accumulation of iron was detected in the livers of animals, which were treated with sMLs instead of free USPIOs. Taken together, our long-term MRI investigations revealed that free USPIOs and sMLs display a similar temporal tissue distribution. Both preferentially accumulated in the liver, whereas sMLs seemed to get cleared more slowly. Here, it has to be mentioned that with MRI imaging and
ICP-OES quantification, we cannot distinguish between intact USPIO-loaded sMLs and USPIO nanoparticles released from liposomes. Although, from our in vitro experiments, we know that more than $75 \%$ of USPIOs remain in the sMLs upon storage for 1 week; the situation in vivo will certainly be different.

\section{Conclusion}

The purpose of our study was to synthesize and characterize sMLs with adequate properties for MRI using commercially available USPIOs. We could demonstrate that not all types of USPIOs are suitable for encapsulation in liposomes. We found that a maximum threshold concentration of USPIOs could be encapsulated into unilamellar liposomes with a mean particle diameter of less than $200 \mathrm{~nm}$. We could also show that this USPIO concentration is sufficient to produce a pronounced signal enhancement in MRI in vitro and in vivo. Taken together, we have established a robust, sterically stabilized ML formulation with efficient MRI contrast properties for functional imaging. Currently, our studies are directed toward the targeting potential of such sMLs to enable their application in diagnostic medicine, with a specific focus on cardiovascular diseases, such as atherosclerosis.

\section{Acknowledgments/disclosure}

We greatly appreciate the help of Dr Helmar Wiltsche (University of Technology, Department of Analytical Chemistry, Graz, Austria) for the ICP-OES measurements.

This work was supported by the Austrian Nano-Initiative, which cofinanced this study as part of the NanoHealth Project, along with the Austrian Research Promotion Agency, as well as the government of Styria (HTI-Call, project identifier LIPOCEST). The authors report no conflicts of interest in this work. 


\section{References}

1. Lasic DD. The mechanism of vesicle formation. Biochem J. 1988;256(1):1-11.

2. Torchilin VP. Liposomes as delivery agents for medical imaging. Mol Med Today. 1996;2(6):242-249.

3. Williams DF. Williams Dictionary of Biomaterials. Liverpool: University Press; 1999.

4. Torchilin V. Multifunctional and stimuli-sensitive pharmaceutical nanocarriers. Eur J Pharm Biopharm. 2009;71(3):431-444.

5. Mulder WJ, Strijkers GJ, van Tilborg GA, Griffioen AW, Nicolay K. Lipid-based nanoparticles for contrast-enhanced MRI and molecular imaging. NMR Biomed. 2006;19(1):142-164.

6. Kelly C, Jefferies C, Cryan SA. Targeted liposomal drug delivery to monocytes and macrophages. J Drug Deliv. 2011;2011:727241.

7. De Cuyper M, Joniau M. Magnetoliposomes. Formation and structural characterization. Eur Biophys J. 1988;15(5):311-319.

8. Martina MS, Fortin JP, Menager C, Clement O, Barratt G, GrabielleMadelmont C, et al. Generation of superparamagnetic liposomes revealed as highly efficient MRI contrast agents for in vivo imaging. J Am Chem Soc. 2005;127(30):10676-10685.

9. Plassat V, Martina MS, Barratt G, Menager C, Lesieur S. Sterically stabilized superparamagnetic liposomes for MR imaging and cancer therapy: pharmacokinetics and biodistribution. Int J Pharm. 2007; 344(1-2):118-127.

10. Sabaté R, Barnadas-Rodriguez R, Callejas-Fernandez J, HidalgoAlvarez R, Estelrich J. Preparation and characterization of extruded magnetoliposomes. Int J Pharm. 2008;347(1-2):156-162.

11. Viroonchatapan E, Sato H, Ueno M, Adachi I, Tazawa K, Horikoshi I. Magnetic targeting of thermosensitive magnetoliposomes to mouse livers in an in situ on-line perfusion system. Life Sci. 1996;58(24): 2251-2261.

12. Soenen SJ, Hodenius M, De Cuyper M. Magnetoliposomes: versatile innovative nanocolloids for use in biotechnology and biomedicine. Nanomedicine (Lond). 2009;4(2):177-191.

13. Gossuin Y, Gillis P, Hocq A, Vuong QL, Roch A. Magnetic resonance relaxation properties of superparamagnetic particles. Wiley Interdiscip Rev Nanomed Nanobiotechnol. 2009;1(3):299-310.

14. Park JY, Daksha P, Lee GH, Woo S, Chang Y. Highly water-dispersible PEG surface modified ultra small superparamagnetic iron oxide nanoparticles useful for target-specific biomedical applications. Nanotechnology. 2008;19(36):365-603.

15. Wu W, Chen B, Cheng J, Wang J, Xu W, Liu L, et al. Biocompatibility of Fe3O4/DNR magnetic nanoparticles in the treatment of hematologic malignancies. Int J Nanomedicine. 2010;5:1079-1084.

16. Di Marco M, Sadun C, Port M, Guilbert I, Couvreur P, Dubernet C. Physicochemical characterization of ultrasmall superparamagnetic iron oxide particles (USPIO) for biomedical application as MRI contrast agents. Int J Nanomedicine. 2007;2(4):609-622.

17. Gupta AK, Gupta M. Synthesis and surface engineering of iron oxide nanoparticles for biomedical applications. Biomaterials. 2005;26(18): 3995-4021.
18. Elias A, Tsourkas A. Imaging circulating cells and lymphoid tissues with iron oxide nanoparticles. Hematology Am Soc Hematol Educ Program. 2009:720-726.

19. Gimi B, Pathak AP, Ackerstaff E, Glunde K, Artemov D, Bhujwalla ZM. Molecular imaging of cancer: applications of magnetic resonance methods. Proc IEEE Inst Electr Electron Eng. 2005;93(4): 784-799.

20. Amstad E, Zurcher S, Mashaghi A, Wong JY, Textor M, Reimhult E. Surface functionalization of single superparamagnetic iron oxide nanoparticles for targeted magnetic resonance imaging. Small. 2009;5(11): 1334-1342.

21. Plaza RC, Arias JL, Espin M, Jimenez ML, Delgado AV. Aging effects in the electrokinetics of colloidal iron oxides. J Colloid Interface Sci. 2002;245(1):86-90.

22. Mulder WJ, Strijkers GJ, Griffioen AW, van Bloois L, Molema G, Storm G, et al. A liposomal system for contrast-enhanced magnetic resonance imaging of molecular targets. Bioconjug Chem. 2004;15(4): 799-806.

23. Uster PS, Allen TM, Daniel BE, Mendez CJ, Newman MS, Zhu GZ. Insertion of poly(ethylene glycol) derivatized phospholipid into preformed liposomes results in prolonged in vivo circulation time. FEBS Lett. 1996;386(2-3):243-246.

24. Klibanov AL, Maruyama K, Torchilin VP, Huang L. Amphipathic polyethyleneglycols effectively prolong the circulation time of liposomes. FEBS Lett. 1990;268(1):235-237.

25. Edwards K, Johnson M, Karlsson G, Silvander M. Effect of polyethyle neglycol-phospholipids on aggregate structure in preparations of small unilamellar liposomes. Biophys J. 1997;73:258-266.

26. Dandamudi S, Campbell RB. Development and characterization of magnetic cationic liposomes for targeting tumor microvasculature. Biochim Biophys Acta. 2007;1768(3):427-438.

27. Dandamudi S, Campbell RB. The drug loading, cytotoxicty and tumor vascular targeting characteristics of magnetite in magnetic drug targeting. Biomaterials. 2007;28(31):4673-4683.

28. Borenstain V, Barenholz Y. Characterization of liposomes and other lipid assemblies by multiprobe fluorescence polarization. Chem Phys Lipids. 1993;64(1-3):117-127.

29. Bowen CV, Zhang X, Saab G, Gareau PJ, Rutt BK. Application of the static dephasing regime theory to superparamagnetic iron-oxide loaded cells. Magn Reson Med. 2002;48(1):52-61.

30. Skouras A, Mourtas S, Markoutsa E, De Goltstein MC, Wallon C, Catoen S, et al. Magnetoliposomes with high USPIO entrapping efficiency, stability and magnetic properties. Nanomedicine. 2011;7(5): 572-579.

31. Weissleder R, Bogdanov AA Jr, Neuwelt EA, Papisov M. Long-circulating iron oxides for MR imaging. Adv Drug Deliv Rev. 1995;16:321-334.
International Journal of Nanomedicine

\section{Publish your work in this journal}

The International Journal of Nanomedicine is an international, peerreviewed journal focusing on the application of nanotechnology in diagnostics, therapeutics, and drug delivery systems throughout the biomedical field. This journal is indexed on PubMed Central, MedLine, CAS, SciSearch $\AA$, Current Contents ${ }^{\circledR} /$ Clinical Medicine,

\section{Dovepress}

Journal Citation Reports/Science Edition, EMBase, Scopus and the Elsevier Bibliographic databases. The manuscript management system is completely online and includes a very quick and fair peer-review system, which is all easy to use. Visit http://www.dovepress.com/ testimonials.php to read real quotes from published authors. 